\title{
0-D STUDY OF THE COMPRESSION OF LOW TEMPERATURE SPHEROMAKS
}

PPPL- -2246

D.D. Meyerhofer, R.A. Hulse, and E. G. Zweibel"

Plasma Physics Laboraiory, Princeton University

P.O. 451 Princeton, NJ 08544

\begin{abstract}
Compression of low temperature spheromak plasmas has been studied with the aid of a O-D two-fluid computer code. It is found that in a plasma which is radiation dominated, the electron temperature can be increased by up to a factor of seven for a compression of a factor of two, provided the temperature is above some critical value $(\sim 25 \mathrm{eV})$ and the electron density particle confinement time product $n_{e} \tau_{p} Z 1 \times 10^{4} / \mathrm{cm}^{3}$. If the energy balance is dominated by particle confinement losses rather than radiation losses, the effect of compression is to raise the temperature as $T_{e} \sim C^{6 / 5}$, for constant $\tau_{p}$.
\end{abstract}

-Permanent address: Dept. of Astrophysical, Planetary, and Atmospheric Sciences, U.of Colorado, Boulder, Co 80309.

\section{DISCLAIMTR}

This report was prepared as an account of work sponsored by an agency of the United States Goverament. Neither the United States Government nor any agency thereof, nor any of their employes, makes any warranty, express or impliod, or assumes any legal liability or responsibility for the eccuracy, completeness, of usefulness of any information, apparalus, product, or process disclowed, or represents that its use would not infringe privately owaed rights. Reference berein to any specific commercial product, process, of service by trade name, trademark, manufactufter, or otberwise does not necessurily corstitute or imply its endorsement, rocommendation, of fayoring by the United States Goverament or any ageacy thereof, The viets and opinions of euthors expreased herein do nat necessarily state or reffect those of the United States Goveroment or any agency thereol. 


\section{INTRODUCTION}

The spheromak $[1,2]$ is a small aspect ratio toroidal plasma with toroidal and poloidal fieid strengths comparable. The magnetic fields are internally generated, with the size of the plasma determined by an externally imposed equilibrium field. This configuration, with no hardware(i.e, toroidal field coils) on the symmetry axis, lends itself to the use of compression for auxiliary heating $[3,4]$.

Spheromak compression has been examined by Katsurai and Yamada [4], and by Jardin[5]. Both papers argued that the spheromak should compress self-similarly, and hence follow the scaling laws for 'Type-A' compression of Furth and Yoshikswa[6]. Katsurai and Yamada assumed that the compression would be adiabatic. Jardin suggested that compression could be used to burn through the low-Z radiation barrier[5]. For tokamak discharges, the possibilities for compression with radiation losses were discussed by Furth[7] and Green et al. [8]. With radiation included, the compression is no longer adiabatic.

The power balance of currently operating splieromaks has been examined $[9,10]$, and for these plasmas the dominant loss terms appear to be impurity radiation and the losses due to finite particle confinement time. These processes are coupled because the confinement time strongly affects the equilibrium cooling rate of the impuritiey[11]. If the replacement time is short, the impurity never 'burns through' to the less radiative Be-like, $\mathbf{H}$-like, and fully Sonized charge states, as the particle losses are offset by the repopulation of the more radiative charge states. The resulting equilibrium cooling rate can thus be many times that found in coronal equilibrium. There is also additional ionization energy, which must be supplied by the plasma.

This letter examines the effect of impurity radiation and finite particle confinement time on the changes in the plasma temperature due to compression. The precompression values of $T_{e}$ and $n_{e} T_{p}$ are found to be important in determining the effectiveness of the compression in raising the plasma temperature.

\section{COMPRESSION SCALING}

If plasma compression is carried out on a time scale short compared to the resistive magnetic diffusion time, magnetic flux is conserved. In addition, if any particles which are lost are assumed to be replaced (perfect recycling), the scaling laws for 'Type-A' compression [6], with $R, a \sim 1 / C$, shown in Table $I$, hold. The stored magnetic energy scales as $C$ (not as $C^{2}$ in Ref. 6). These scalings hold even if nonadiabatic terms are included in the evolution of the plasma kinetic energy.

The evnlution of the plasma temperature is determined by both adiabatic and nonadiabatic terms. If the adiabatic equation of state is assumed (conseryation of entropy), $p \rho^{-\frac{5}{3}}=$ Constant, with $p=n T$ the plasma pressure and $\rho$ the mass deasity, the temperature scales as

$$
T \sim C^{2}
$$


The two adiabatic processes included in the energy balance are the compressional work done on the plasma by the exteralal magnetic field and the loss of electron energy through the ionization of par'icles. The latter term is adiabatic in the sense that in the system of ions, electrons, and neutrals, energy is conserved as long as all the particles remain within the system. The additional degrees of freedoin associated with the ionization can change $\gamma$, the ratio of the specific heats, if the number of ionizations occurring is large[12]. In practice, this is not a large effect, if one assumes that the initial hydrogen plasma is fully ionized, and there are only a ferw percent impurities.

The nonadiabatic contributions to the plasma power balance include ohmic hearing, resulting from the decay of the magnetic fields, impurity radiation, and the losses due to Ginite particle confinement time.

When it is assumed that the radiation losses in the plasma are insignificant, the ohmic heating must balance the loss due to the finite particle confinement time. Thus, for fixed $\tau_{p}$, the electron temperature scales under compiession as

$$
T_{e} \sim C^{\frac{8}{5}}
$$

Because scaling of the observed particle confinement times are unknown[9], it will be assumed that the confinement time remains constant during compression, consistent with the scaling of the classical particle confinernent time under adiabatic compression.

In a plasma with impurity radiation as the dominant loss term, the increased density due to compression has two major effects on the power balance of the plasma. Initially, the radiated power density increases, $P_{\text {rod }} \sim n_{\varepsilon}^{2} \sim C^{6}$, compared to the scaling of the ohmic heating power, $P_{\text {oh }} \sim C^{3}$ for adiabatic compression and $P_{\text {oh }} \sim C^{6}$ for constant temperature compregeion. Thus, a plasma with an initial balance between ohmic heating and radiation losses might have to electron te perature increase due to compression.

A competing effect of increased density due to compression is that the collisional ionization rate per ion increases and can lead the impurities to evolve to less radiative charge states. An examination of the paper by Carolan and Piotrowicz[11](herearter referred to as CP) suggests when this effect allows compression to be effective in radiation-dominated plasmas. In $C P$, the radiation rate coefficients $\left(W-\mathrm{cm}^{3}\right)$ for oxygen and carbon are plotted versus temperature for various values of $n_{e} \tau_{p}$, with $n_{e} \tau_{p}=1.0 \times 10^{12} \mathrm{~s} / \mathrm{cm}^{3}$ close to coronal equilibrium. Above some critical temperature, roughly $25 \mathrm{eV}$ for oxygen and $20 \mathrm{eV}$ for carbon, the radiated power function decreases with ilcreasing $n_{e} \tau_{p}$, from $n_{e} \tau_{p} \sim 1.0 \times 10^{9} \mathrm{~s} / \mathrm{cm}^{3}$ to $n_{e} \tau_{p} \sim 1.0 \times 10^{12} \mathrm{~s} / \mathrm{cm}^{3}$. The amount of decrease is such that it keeps the power radiated per ion roughly constant as the electron density is changing, if the particle confinement time and electron temperature remain constant. In this condition, the radiated power density scales as $P_{\text {rad }} \sim C^{3}$, which allows the temperature to scale as $T_{e} \sim C^{2}$. This iucrease in the temperature reduces the radiated power, and the increase in $n_{\varepsilon} \tau_{p}$ also raises $Z_{\text {eff }}$ so that the temperature can rise further. Below the critical temperature, an increase in $n_{e} \tau_{p}$ does not chasge the radiated power function. Thus, in a case where radiative power loss is the dominant loss mechanism, these estimates suggest that if $n_{e} \tau_{p} \gtrsim 1.0 \times 10^{9} \mathrm{~s} / \mathrm{cm}^{3}$ and the uncompressed electron temperature is greater than $25 \mathrm{eV}$ for oxygen or $20 \mathrm{eV}$ for carbon. cornpression can raise the electron temperature significantly. 
To study the interaction among the various terms in the plasme power balance, a zero-dimensional, two-fluid computer code has been written. The most important effects neglected in this treatment are those associated with the plasma projile. The extension to a one- or one and one half-dimensional calculation would be much more complicated, and would require many more assumptions. The $0-D$ calculation can be thought of as a rough average over the central region of the plasma.

The power loss terms incluied in the $0-D$ code are impurity radiation, the loss due to finite particle confinement time(including the power to ionize the replacement particles), and the power lost through the ionization of the impurity ions. Other processes could also be included, i.e., thermal conduction, but their scalings are unknown and the basic physics can be shown with the above terms.

The compressional work done on the electrons, ions, is

$$
P_{c e, i}=-p_{e, i} \frac{1}{V} \frac{\partial V}{\partial t}
$$

where $p_{e, i}$ is the election, ion pressure, and $V$ is the plasma volume. It is assumed that the compression will be driven by a capacitor bank into an inductive vertical feld coil. If resistive effects are neglected, the current in the coil will have the form $I_{v} \sim I_{0} \sin w t$, with $\omega^{\prime}=\pi / 2 \tau_{\mathrm{c}}$. The size of the plasma is assumed to be determined by

$$
B_{v} \propto \frac{I_{v}}{R}
$$

The effect of an increase of the size of the plasma is $C^{2} \sim B_{v}(t) / B_{v 0}$. Thus, the time evolution of the plasma volume due to compression is

$$
\frac{1}{V} \frac{\partial V}{\partial t}=-\frac{3}{2} \frac{\left(C_{0}^{2}-1\right) \omega \cos \omega t}{\left(1+\left(C_{0}^{2}-1\right) \sin \omega t\right)}
$$

The time evolution of the current density inciudes the compressional effect as well as the resistive decay.

The radiated power is calculated through a time dependent nonequilibrium coronal calculation of the fraction of the impurity density in the various charge states. The impurity ionization, recombination, and radiated power coefficients are calculated using a set of subroutines degcribed in Refs. 13-15. In aduition, the important effects of a finite impurity confinement time are included. Atomic processes inciuded are collisional ionization, radiative and dielectronic recombination, and charge exchange recombination. For the latter, an estimate of the neutral density is obtained from $\tau_{p}$. For an equilibrium density, the particle source and loss rates must be equal, $n_{H} / \tau_{p}=n_{0} n_{e}(a v\rangle_{i o n}$, where $n_{0}$ is the neutral density. and $\langle\sigma v\rangle_{\mathrm{im}}$ is the collisional ionization rate of hydrogen. Charge exchange recombination is found to have a negligible effect on the imputity ionization state balance for the cases studied.

The fraction of impurities in various charge states,

$$
f_{+q}=\frac{n_{i m p}^{+q}}{\sum_{q=0}^{Z} n_{i m p}^{+q}},
$$


can be solved for through a coupled set of rate equations,

$$
\frac{\partial f_{q}}{\partial t}=I_{q-1} f_{q-1}+R_{q+1} f_{q+1}-\left(I_{q}+R_{q}\right) f_{q}-\frac{f_{q}}{t_{p}}+S_{q}
$$

for $0 \leq q \leq Z$, with $Z$ the atomic number of the impurity, $I_{q}, R_{q}$ are the ionization and recombination rate coefficients per ion.

The complete $0-D$ problem is described by two sets of coupled equations, one describing the evolution of the impurity fractions, another describing the evolution of the other plasma parameters. These two sets are solved on alternate time steps. This allows the rate coefficients to be calculated once for each step advancing the impurity fractions. All of the computational parameters, for example the time step for the implicit techniques and the interpolation range of the rate coefficients, were varied to minimize the computational time while leaving the results unchanged.

\section{NUMERICAL RESULTS}

The efiect of compression on the electron temperature is discussed here for two extrerpe cases, one with the dominant power loss due to finite particle confinement time, and the other where impurity radiation is the dominant loss. The effectiveness of the compression is measured by $\Gamma$, the ratio of the peak electron temperature of the compressed plasma to that in the uncompressed plasma. $\Gamma=4$ for adiabatic compression with $C=2$.

In the cases where finite particle confinement time is the dominant loss term, Eq. (2) gives $\Gamma=2.3$ for a compression with $C=2$. This figure is obtained as well in the numerical results.

The more interesting case is when impurity radiaticil power is the dominant loss mechanism. For definiteness, it is assurned that oxygen is the only impurity species, and the results presented are for $C=2$.

Figures $1 \mathrm{a}$ and $\mathrm{lb}$ show the evolution of the electron temperature and electron power balance for plasma where the power lost through impurity radiation, $P_{\text {rad, }}$, dominates that die to finite particle confinement time $P_{\text {le. }}$. The initial conditions are $j=125 \mathrm{~A} / \mathrm{cm}^{2}$, $n_{i}=4 \times 10^{13} \mathrm{~cm}^{-3}, \tau_{p}=1.0 \times 10^{-3} \mathrm{sec}$, and $3 \%$ oxygen. Figures $1 \mathrm{c}$ and $1 \mathrm{~d}$ show the effect of compression from 0.5 to $0.6 \mathrm{msec}$ on the electron temperature and power balance. $P_{c e}$ is the compressional input energy, and $P_{i e}$ is the power lost by the electrons to the ions. In this case $\Gamma=6.4$, so that the electron temperature bas increased more than would be expected in adiabatic compression. The precompression temperature is $28 \mathrm{eV}$ s.s it is expected from an eramination of CP that the compression should be effective in raising the electron temperature in this case.

A series of simulations were run, keeping $n_{e} \tau_{p}=4 \times 10^{10} \mathrm{sec} / \mathrm{cm}^{3}$ and with $5 \%$ oxygen. with the precompression electron temperature varied by changing the current density. The effectiveness of compression $(\Gamma)$ is platted in Fig. 2 versus precompression temperature. It can be seen that, as expected from CP, the compression is effective if the precompression temperature exceeds $\sim 25 \mathrm{eV}$. 
The effect of the precompression $n_{e} \tau_{p}$ on $\Gamma$ is shown in Fig. 3. In this case, the ohmic heating per particle was kept roughly constant, and the impurity fraction was varied so that the uncompressed plasmas would be dominated by radiation losses and have precompression electron temperature of roughly $30 \mathrm{eV}$. As expected from $\mathrm{CP}$, the compression was not effective if the density confinement time product was less than $3 \times 10^{9} \mathrm{sec} / \mathrm{cm}^{3}$. A similar set of runs was made with the precompression temperature roughly $20 \mathrm{eV}$, and for all values of $n_{e} \tau_{p}$, the compressed electron temperature remained at $20 \mathrm{eV}$.

Simulations were also performed with carbon as the impurity species, and the results were found to agree with the expectations obtained from CP. If the precompression temperat ure is above $20 \mathrm{eV}$ in a carbon radiation-cusinated discharge and $n_{e} T_{p} \gtrsim 1.0 \times 10^{9} \mathrm{sec} / \mathrm{cm}^{3}$, the compression is effective in significantly raising the electron temperature.

The radiated power functions of higher $Z$ elements, such as iron, show less dramatic but still useful decreases in the radiated power function with increasing $n_{e} \tau_{p}[11]$. Simulations were run with iron, and again the discharges with $P_{\text {rad }} \gg P_{l e}$ showed large increases in the electron temperature, reaching $\Gamma \geq 4$.

For cases with a balance of losses due to impurity radiation and finite particle confinement time, the increase in electron temperature is found to be between that of either of the two extremes.

\section{CONCLUSION}

The compressioe of low temperature spheromak plasmas has been examined and simulated for a vide range of parameters with a time-dependent power balance conputer model. It was found that the most important effect of the compression is to increase the current density and electron density, which allows higher temperature states to be achieved. The greatest improvement occurs for states where the radiation power dominates the power loss due to particle recycling. An examination of Carolan and Piotrowicz[11] allows an estimate of required plasma parameters for compression to be effective in raising the eiectron temperature. A radiation-dominated plasma with oxygen as the dominant impurity must have a central electron temperature greater than $25 \mathrm{eV}$, while if carbon is the dominant impurity species, the temperature must be greater then $20 \mathrm{eV}$. In both cases, the electron density recycling time product, $n_{e} \tau_{p}$, must be greater than $1.0 \times 10^{9} \mathrm{~s} / \mathrm{cm}^{3}$. Once the effects governing the particle confinement can be better estimated, a one- or one and one half-dimersional simulation of spheromak compression could be used to provide a more detailed examination of the compression physics.

\section{Acknowledgments}

We wish to thank R.J. Goldston, M. Yamada, and S. Jardin for many useful discussions. This work is supported by the U.S. Department of Energy Contract No. DE-AC02-76CHO3073. 


\section{References}

[1] Rosenblu:ì, M.N., Bussac, M.N., Nurl. Fusion 19 (1979) 489.

[2] Bussac, M.N., Furth, H.P., Okabayash: M., Rosenblıth, M.N., Todd, A.M., Presented at the 7th Int. Conf. on Plasma Physics and Controlled Nurlear Fusion, Inusbruck, Austria(1978).

[3] Yamada, M., S-1 preiiminary concept(1979](unpublished).

[4] Katsurai, M., Yamada, M., Nucl. Fusion 22 (1982) 1407.

(5) Jardin, S.C., Presentec at the 6th U.S. Symposium on Compact Toroid Research, Princeton, NJ(1984).

[6] Furth, H.P., Yoshikawa, S., Phys. Fluids 13 (1970) 2593.

[7) Furth, H.P., presented at the 3rd Int. Symp. On Toroidal Plasma Confinement, Garching(1973), paper B-9-1

[8] Green, B., Noll, P., Sheffeld, J., J. Plasma Phys. 17 (1975) 1101.

[9] Barnes, C.W., Jarboe, T.R., Hoida, H.W., Wright, B.L., Los Alamos Laboratory Report LA-UR-843667(1984) submitted to Nucl. Fusion.

[10] Meyerhofer, D.D., Goldston, R.J., Hart, G.W., Levinton, F.M., Mimura, M., Yamada, $M$, in the Proc. 7th Compact Toroid Conference, Santa Fe, NM(1985).

[11] Carolan, P.G., Piotrowica, V.A., Plasma Phys. 25 (1983) 1065.

[12] Cox, J.P., and Guili, R.T., Principles of Stellar Structure, Gorron and Breach, NY, (1978), vol. 1

[13] Hulse, R.A., Nucl. Technol./Fusion 3 (1983) 259.

(14) Post D.E., Jensen, R.V,, Tarter C.B., Grasberger, W.H., and Lokke, W.A., Atomic Data and Nuclear Data Tables 20 (1977) 397.

[15] Hulse, R.A., Post, D.E., Mikkelsen, D.R., J. Phys. B: 13 (1980) 3895. 
Adiabatic Scalings

$\overline{\text { Plasma Current } \quad I \sim C}$

Current Density $\quad j \sim C^{3}$

Plasma Density $\quad n_{\varepsilon} \sim C^{3}$

Magnetic Field $\quad B_{t} \sim B_{\mathrm{p}} \sim C^{2}$

Table 1: Scalings of various plasma quantities under a compression of a factor $C$, where $R \sim 1 / C$. 
FIG. 1. Evolution of the electron temperature and power balance for an uncompressed plasma $(\mathrm{a}, \mathrm{b})$, and for the same plasma undergoing a factor of 2 compression, beginning at $5 \times 10^{-4} \mathrm{sec}$ and ending at $6 \times 10^{-4} \mathrm{sec}$. (c,d).

FIG. 2. Ratio of the compressed to uncompressed electron temperature( $\Gamma)$ for a factor of two compression for a series of uncompressed temperatures. These cases have $n_{e} \tau_{p}=4.0 \times 10^{10} \mathrm{~s} / \mathrm{cm}^{3}$ and $5 \%$ oxygen. The uncompressed temperature was varied by varying the current density.

FIG. 3. Post-compression electron temperature versus $n_{e} \tau_{p}$ for a series of simulations, where the ohmic heating per electr'n was beld fixed, the impurity fraction was varied to give initial temperatures if $\sim 30 \mathrm{eV}$, and roughly $70 \%$ of the input power lost through impurity radiation. 

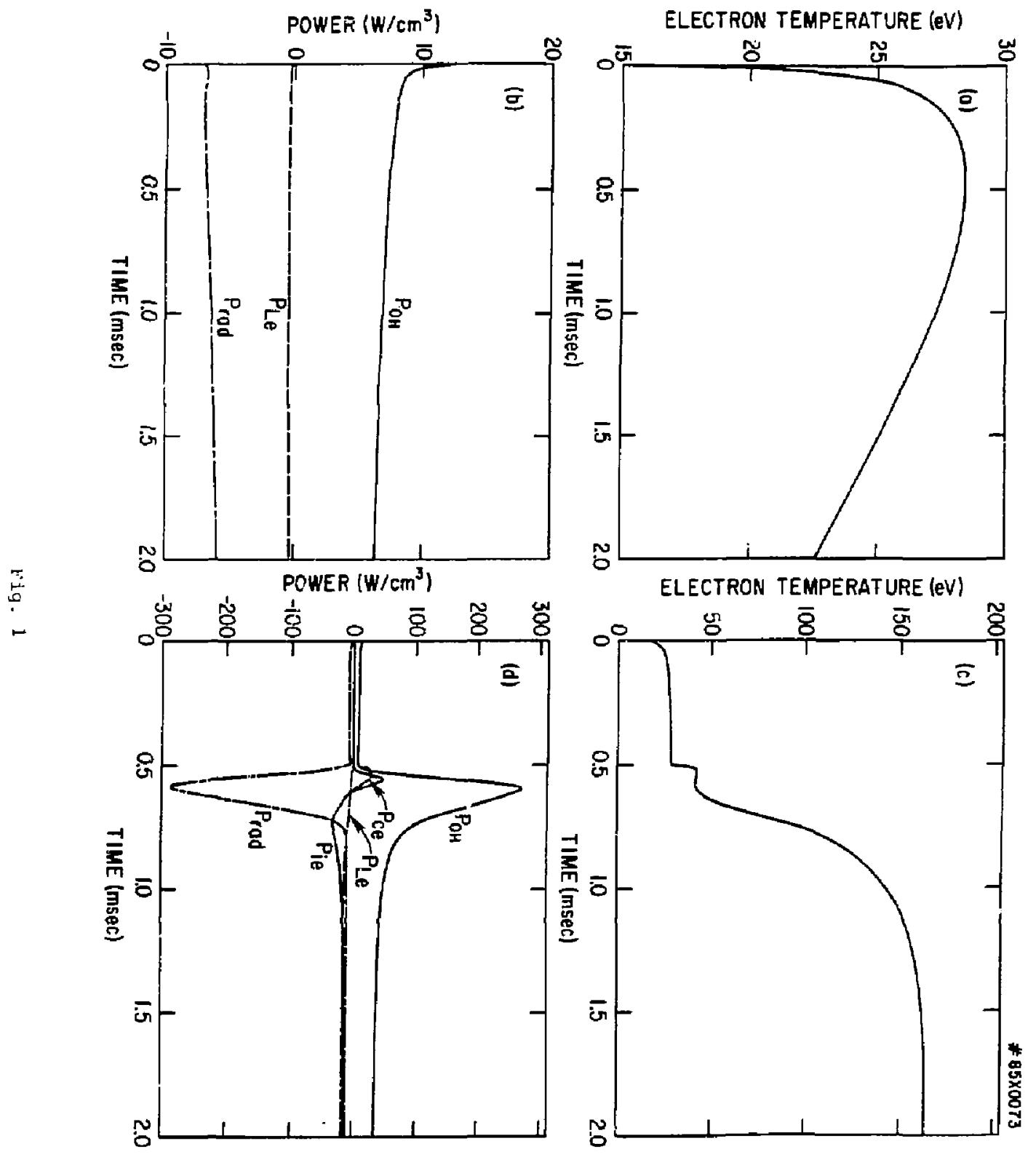
吕

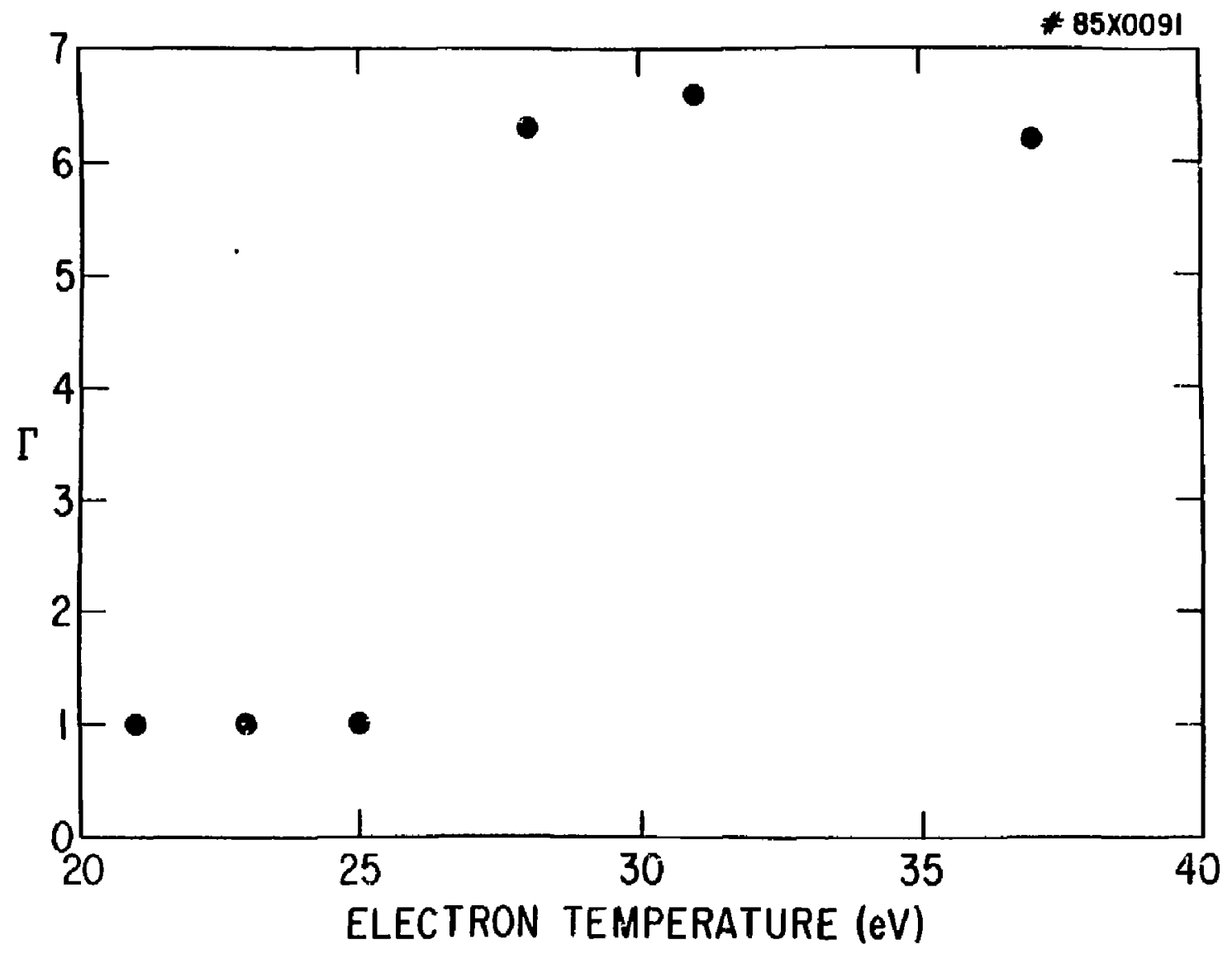

Fig. 2 


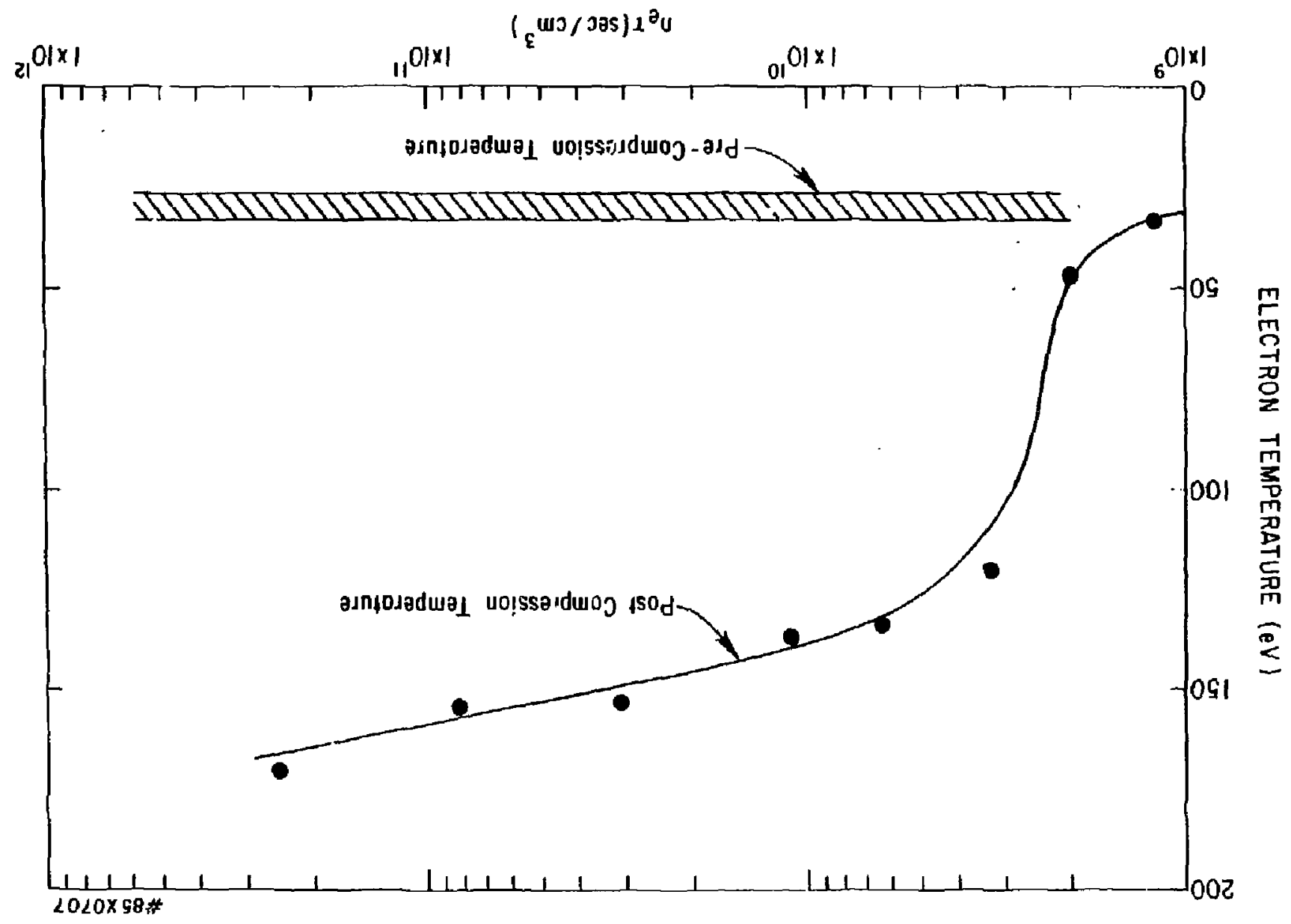


Plastra Res Eab, Austra Nat'I Univ, ALSTRALCA

Dx. Frank J. Paolioni, Iniv of tollongong, ALSTRALIA

Prof. I.R. Jones, Flinders Uni-y., AlSTRALIA

Prof. M.H. Tennan, Thiv Syaney, AUSTRALta

prof. F. Cap, Inst theo Phys, acsigra

Prof. Frark Verhaest, Inst theoretische, BingIIM

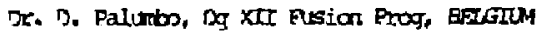

Fole rogle Militain, Lab de thys Plames, BEGTM

DI. P.H. Sakanaka, Ohiv matahal, EATHL

DT. C.R. James, thiv of Alberta, Carana

prot. J. Teidman, Iniv of Nentreal, CANpDA

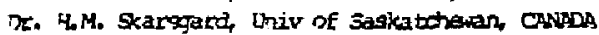

Prof. S.R. Smenivagan, University of Calgary, CANaDA

Prof. Nrior $w$. Johnstor, INES-Energie, CALDA

Di. Hannes Bamard, Univ Rritish Colunbia, CANADA

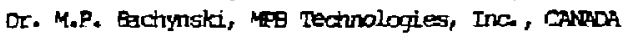

Chaik River, Nucl Iab, CAlapA

mengur Ii, Sw Inst ghysics, CTRA

inbrary, Ising tha University, oma

Liblarian, Instibute of Frysics, Gma

Inst Plasta Ehys, Academia Sinica, Grma

ix. peter Lukac, Kamensicho Univ, Czromosrorkta

The Libcarian, Cullatin Labaratory, ExEAND

Prof. Schatzan, Joserataice de $\mathrm{Ni}$.e, FRAMCE

J. Radet, Car-EF, FRANCE

aM Dupas Eibrary, AM Dupas Iibrary, FRACE

DF. Ton threl, Rcadeny Bibliographic, Haxg xaNG

Preprint Library, Cent Reg tnst Ptys, Hurearx

7T. S.K. Trehan, Parjah University, DDIA

Dr. Indra bhan Ial Das, Eanaras Himu Univ, INOLA

DT. I.K. Chavia, south Grjarat Univ, INSTA

Dr. R.K. Chajajlani, Vikran Univ. Fora

7T. B. Dasgluta, Saha Inst, INDIA

DT. P. Kar. Ptyyical Researcil Lab, INDIA

rc. Phillip Rosenal, Israel Inst Tech, ISRAEL

PLof. S. Oupermen, Tel Audv inivergity, ISRAES

Prof. G. Rogtagni, Oniy di Padova, TIMLY

Librarian, Int'l Ctr Theo Ptys, ITALY

- tiss Clelia De Palo, Assoc ElRATOM-2NEA, TIATY

Biblioteca, del ONR ERATOM, ITRY

Dr. H. Yanato, Toshihe Res a Dev, JAEss

Direc Dept ig. Tolzmak DeN. JARR, , TRAN

Prof. Wholyut Inove, University of Tokyo, JAFAN

pesearch Info Center, Nagoya thiversity, JAPAN

Exof. KYoji Nishikan, Univ of Hiroshima, JAPAN

Erof. Sigena Mori, JRFI, TrSaN

ilbrary, Kyoto University, JAPAN

Pmot. Ichiro Kawakemi, Nihon Univ, JAPAN

Prof. Satoghi Itoh, Rin!shas liaiversity, JAPAN

DT. D.I. Thoi: adv. Inst Sci \& Thch, KDREA

Teth Info Mivision, KAERT, KDPEA

Fibliothex, Em-Inst voor Plagra, NETHERLANDS
Frof. B.S. LiIey, rniversity of haikator Nal ZEALAND Erof. J.A.C. Cabral, Inst Superior Terh, PORTSG C Or. Octavian eetons, ALI JJZA Uni rersily, ROMANIA Frof. M.A. Hellberg, University of Natel, SO AFrICA

Dr. Johan de villiers, Plasma zhysics, Mxcor, SO AFsica Eusion Di.v, Libracy, JPN, SPAIN

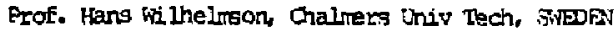

Dc. Lennart Stenflo, University of UMA, SWIDDN

Library, Roynil Inst Tech, SweDes

Centre de Recherchesen, Eroile Polytech Fed, SWITERTivo

Dr. V.T. Tolok, Whadkou Phys Tech Ins, [SSRR

Dr. D.D. Ryutor, Slberian Acar 5Ci, US5R

Dr. G.A. Elises, kurchatov Institute, isst

Dr. V.A. Glukhikh, Inst Electro-physical, iSSR

Instibute Gen. Physics, USSR

prof. T.J.M. Eoyri, Univ college $v$ iales, whiES

Dr. $k$, schindlar, outr Univarsicat, w. Gowasy

Buclear Res Fstab, Julich Lta, Wh GrimanY

Librarian, Max-Plandk Instiut, W. GFMANY Sibliothel, Inst Plasmaforschung, w. GDMANY Prof. R.X. Janev, Inst thys, YugDSLAVIA 